\title{
Propagation of optical vortices in loop resonators on the basis of multimode optical fibers
}

\author{
C. Alexeyev ${ }^{1}$, E. Barshak ${ }^{1}$, D. Vikulin ${ }^{1}$, B. Lapin ${ }^{1}$, M. Yavorsky ${ }^{1}$ \\ ${ }^{1}$ V.I. Vernadsky Crimean Federal University, Prospekt Vernadskogo 4, Simferopol, Russia, \\ 295007
}

\begin{abstract}
In this paper we theoretically study the propagation of optical vortices (OVs) through a loop resonator (LR) on a multimode optical fiber. We demonstrate the presence in LRs of a resonance, which is in the inversion of the topological charge (TC) of the transmitted OV. We show that in the outcoming field the weights of the OVs with opposite TCs may be sensitive to wavelength variations of the LR's length. Near the resonance points this property can be used for super-efficient control over the TC and orbital angular momentum of the outcoming field. We discuss application of this effect for temperature sensing. We also demonstrate the resonance power spikes in the loop and show that the resonance loop fields are the Hermite-Gaussian modes. In addition, we study the spectral characteristics of OV transmission and show feasibility of LR-based comb filters for OVs.
\end{abstract}

\section{Introduction}

Since the first experimental demonstration of the fiber loop resonator (LR) [1] its application potential has been realized to a wide extend. The similarity of this system's comb transmission spectrum to the one of the scanning Fabry-Perot interferometer makes LRs useful tools in optical-filter and spectrum-analyzer applications [2,3]. The spectrum's sensitivity to environmental conditions enables creation of various LR-based temperature $[4,5]$, chemical [6], refractometric [7] and displacement [8] sensors. Researches have also confirmed that LRs can be useful for nonlinear optics, in particular, for third [9] and second [10] harmonic generation.

The physical mechanism of an evanescent coupling that underlies operation of LRs is also of key importance in other fiber optical devices similar in performances to LRs: microcoil [11], knot [12] and ring [13] resonators. Widening the variety of fiber-based resonators entailed expanding of application range of such devices. Currently, such systems are considered as basic elements for optical communication [14], engineering photonic delay lines [15], add-drop filters [16] and microwave generation [17]. Recently, some new trends have emerged connected with topological phases in resonator arrays [18], nanoparticle sensing [19] and graphene-incorporating resonators [20].

Until quite recently, the studies of fiber resonators, as well as related question of light propagation in coupled fibers, were limited to monomode fibers. Advances in optical communications via orbital angular momentum (OAM) states [21] and increasing application of fiber optical vortices (OVs) [22-27] makes it topical to fill up the gap in our knowledge of LRs operation on multimode fibers. To date, a few papers studied evolution of OVs in coupled fibers addressing, in particular, vortex states cloning [28] and OAM tunneling [29] in them. In the only paper on OV's evolution in coil resonators it have been outlined general principles of solving related problems and shown the inversion of topological charge (TC) [30].

In this paper we apply the previously developed formalism [27,31] to study the OV transmission through a LR. We show that in certain points a special TC-flip resonance takes place, at which the TC of the transmitted 
OV gets inverted. We show that by changing the LR's length parameters one can control in the outcoming field the weights of the OVs with opposite TCs. Those weights can be sensitive to wavelength and even ultrasubwavelength variations of the LR's parameters, in this way enabling super-efficient control over OAM and the TC of the outcoming field. We also discuss possible sensor application of this property. Additionally, we show the existence of resonance power spikes in the loop fields, which might be useful for nonlinear applications. We also study the spectral characteristics of OV transmission in LRs and show feasibility of comb filters on the basis of the effect of OV's power oscillations.

\section{Loop resonator}

The scheme of the LR is shown in Figure 1a. In the boxed area the lower (L) and the upper (U) parallel parts of the multimode fiber are evanescently coupled and form a quadripole of a kind (Figure 1b), which can be considered as a LR, if its port 3 is connected by loop to the port 2 (Figure 1c). In the boxed area the fibers are coupled to each other.
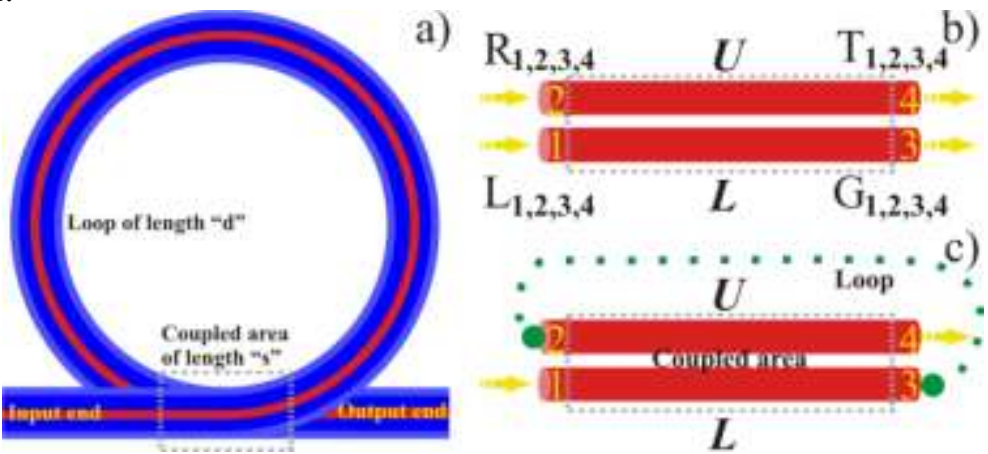

Figure 1. a) A scheme of the LR; in the marked area the parts of the LR are coupled to each other via evanescent fields. b) A quadripole made of two coupled fibers (marked by "U" and "L"). Ports 1, 2 and 3, 4 are the input and output ports, correspondingly. c) An equivalent scheme of the LR based on the quadripole with the bridged ports.

It is known that in the coupling area the fiber field exists in the form of coupled modes, which exact expressions can be found in [32]. However, if the coupling area is not stretched (which is the case for such devices), one can limit oneself to the scalar approximation expressions obtained in [30]. Adapting them to our geometry one can express the normal modes $\psi_{i}$ of the coupled multimode fibers as

$$
\begin{aligned}
& \left|\psi_{1}\right\rangle=|1, L\rangle+|2, L\rangle+|1, U\rangle+|2, U\rangle,\left|\psi_{2}\right\rangle=|1, L\rangle-|2, L\rangle+|1, U\rangle-|2, U\rangle, \\
& \left|\psi_{3}\right\rangle=|1, L\rangle+|2, L\rangle-|1, U\rangle-|2, U\rangle,\left|\psi_{4}\right\rangle=|1, L\rangle-|2, L\rangle-|1, U\rangle+|2, U\rangle, \\
& \left|\psi_{5}\right\rangle=|4, L\rangle+|3, L\rangle+|4, U\rangle+|3, U\rangle,\left|\psi_{6}\right\rangle=|4, L\rangle-|3, L\rangle+|4, U\rangle-|3, U\rangle, \\
& \left|\psi_{7}\right\rangle=|4, L\rangle+|3, L\rangle-|4, U\rangle-|3, U\rangle,\left|\psi_{8}\right\rangle=|4, L\rangle-|3, L\rangle-|4, U\rangle+|3, U\rangle .
\end{aligned}
$$

Here we use the following designation for partial fields in (1):

$$
\begin{aligned}
& |1, L / U\rangle=\exp \left(i l \varphi_{L / U}\right) F_{L / U}\left(r_{L / U}\right) \operatorname{col}(1, i),|2, L / U\rangle=\exp \left(-i l \varphi_{L / U}\right) F_{L / U}\left(r_{L / U}\right) \operatorname{col}(1, i), \\
& |3, L / U\rangle=\exp \left(-i l \varphi_{L / U}\right) F_{L / U}\left(r_{L / U}\right) \operatorname{col}(1,-i),|4, L / U\rangle=\exp \left(i l \varphi_{L / U}\right) F_{L / U}\left(r_{L / U}\right) \operatorname{col}(1,-i),
\end{aligned}
$$

where subscripts " $L$ " and " $U$ " stand for "L-fiber localized" and "U-fiber localized", coordinates $\left(r_{L / U}, \varphi_{L / U}\right)$ are polar coordinates referred to the L-fiber and U-fiber, correspondingly. It should be noted that expressions (2) are written in the basis of linear polarization. The function $F_{l}$ is the radial function [33]. Here $l$ designates TC of the vortices $|1-4, L / U\rangle$. The propagation constants of these normal modes can be expressed as $\beta_{i}=\tilde{\beta}_{l}+\delta \beta_{i}$, where $\tilde{\beta}_{l}$ is the scalar propagation constant and the corrections can be expressed as

$$
\delta \beta_{1,2}=\left(C_{l} \pm D_{l}\right) / 2 \tilde{\beta}_{l}, \delta \beta_{3,4}=-\left(C_{l} \pm D_{l}\right) / 2 \tilde{\beta}_{l}, \delta \beta_{5,6}=\delta \beta_{1,2}, \delta \beta_{7,8}=\delta \beta_{3,4} .
$$

The coupling constants $C_{l}$ and $D_{l}$ determine probabilities for an OV of TC $l$ to tunnel between the fibers without or with charge inversion [32]. 
The above expressions enable one to determine the action of a fiber quadripole onto the incoming field $\psi_{\text {in }}$. Indeed, let this field be the sum of L/U-localized OVs:

$$
\left|\psi_{\text {in }}\right\rangle=L_{1}|1, L\rangle+L_{2}|2, L\rangle+L_{3}|3, L\rangle+L_{4}|4, L\rangle+R_{1}|1, U\rangle+R_{2}|2, U\rangle+R_{3}|3, U\rangle+R_{4}|4, U\rangle,
$$

where $L_{i}$ and $R_{i}$ are some known coefficients. Then we have at the left border of quadirpole:

$$
\left|\psi_{\text {in }}\right\rangle=\sum_{1}^{8} \alpha_{i}\left|\psi_{i}\right\rangle
$$

where $\alpha_{i}$ are unknown decomposition coefficients and $s$ is the coordinate along the coupled area. At the right border of the quadripole the outcoming field is:

$$
\left|\psi_{\text {out }}\right\rangle=\sum_{1}^{8} \alpha_{i}\left|\psi_{i}\right\rangle \exp \left(i \beta_{i} s\right),
$$

where $s$ is the length of the coupled area (see Figure 1). On the other hand, the outcoming from the quadripole field can be presented in the following form:

$$
\left|\psi_{\text {out }}\right\rangle=G_{1}|1, L\rangle+G_{2}|2, L\rangle+G_{3}|3, L\rangle+G_{4}|4, L\rangle+T_{1}|1, U\rangle+T_{2}|2, U\rangle+T_{3}|3, U\rangle+T_{4}|4, U\rangle,
$$

where $G_{i}$ and $T_{i}$ are amplitudes of the outcoming optical vortices. To make from the quadripole the LR one should link the ports 2 and 3 (Figure 1c). Then the OVs with the amplitudes $G_{i}$ enter the loop and go to the port 3 acquiring an additional phase. Due to the scalar approximation we assume that in the loop all vortices have the same propagation constant $\tilde{\beta}_{l}$ and thus the same additional phase:

$$
G_{i} \exp \left(i \tilde{\beta}_{l} d\right)=R_{i}
$$

where $d$ is the loop's length of the LR. Equations (1)-(8) allow one to obtain the system in the unknown coefficients $G_{i}, T_{i}$.

\section{Propagation of optical vortices in loop resonators}

Let us now study propagation of the OVs $|1, L\rangle$ and $|2, L\rangle$ in the LR. Here we choose the OVs only with the left circular polarization, because the LR in the scalar approximation does not affect the state of polarization of the incoming field. Solving the system in the coefficients $G_{i}, T_{i}$ one can obtain the following expressions:

$$
e^{-i \Psi} T_{1,2}=I_{+} \exp \left(i \Phi_{1}\right) \pm I_{-} \exp \left(i \Phi_{2}\right), e^{-i \Psi} G_{1,2}=I_{+} R_{1} \pm I_{-} R_{2},
$$

where $I_{ \pm}=\left(L_{1} \pm L_{2}\right) / 2, \tan \Phi_{i}=\cos \chi /\left(\sin \chi+\sin \gamma_{i}, R_{i}=\cos \gamma_{i} /\left(1-i \exp (i \gamma \sin \gamma)_{i}, \Psi=s \tilde{\beta}_{l}\right.\right.$ and $\gamma_{i}=s \delta \beta_{i}$. Here we assume, that $\left|L_{1}\right|^{2}+\left|L_{2}\right|^{2}=1$ (the power of the incoming field is unity). Also, the condition $\left|G_{1}\right|^{2}+\left|G_{2}\right|^{2} \neq 1$ can take place. It should be noted, that $G_{3,4}=T_{3,4}=0$, because these coefficients refer to the fields with the right circular polarization. For simulations we take: $l=1$, optical contrast $\Delta=0,001$, wavelength $\lambda=632,8 \mathrm{~nm}$, core's refractive index $n_{c o}=1,5$ and radius $r_{0}=8 \lambda, V=3,37$, $C_{l}=-6,39 \cdot 10^{9} \mathrm{~m}^{-2}$ and $D_{l}=-9,98 \cdot 10^{9} \mathrm{~m}^{-2}$.

Consider first the case, when the input port 1 is excited by the OV $|1, L\rangle$, that is $L_{1}=1$ and $L_{2}=0$. Then for the outcoming fields the powers $T_{+}=\left|T_{1}\right|^{2}, T_{-}=\left|T_{2}\right|^{2}$ of the OVs $|1, U\rangle$ and $|2, U\rangle$, correspondingly, can be written as:

$$
T_{+}=\cos ^{2}\left(\Phi_{1}-\Phi_{2}\right), T_{-}=\sin ^{2}\left(\Phi_{1}-\Phi_{2}\right) .
$$

As has been shown [30], conversion takes place only at a sufficient length of the coupling zone. Fig. 2a shows general dependence of $T_{+}$on the coupling length. As is seen, this plot features small-scale variations of transmission amplitude on the background of a large-scale evolution. The scale of the latter is set by the value of coupling constants. Also, unless $s$ is sufficiently large, no effective conversion of $|1, R\rangle \mathrm{OV}$ into $|2, R\rangle \mathrm{OV}$ is possible. In the areas of fully developed oscillations (Figure 2b) on the background of wavelength-scale oscillations there are sharp subwavelength peaks of the transmission coefficient. This property opens new 
possibilities in super-efficient all-fiber control of OAM and TC of OVs. The reason for such wavelength-scale variations is the multibeam interference of OVs with the same TC, which repeatedly tunnel into the loop in the coupling segment [31].
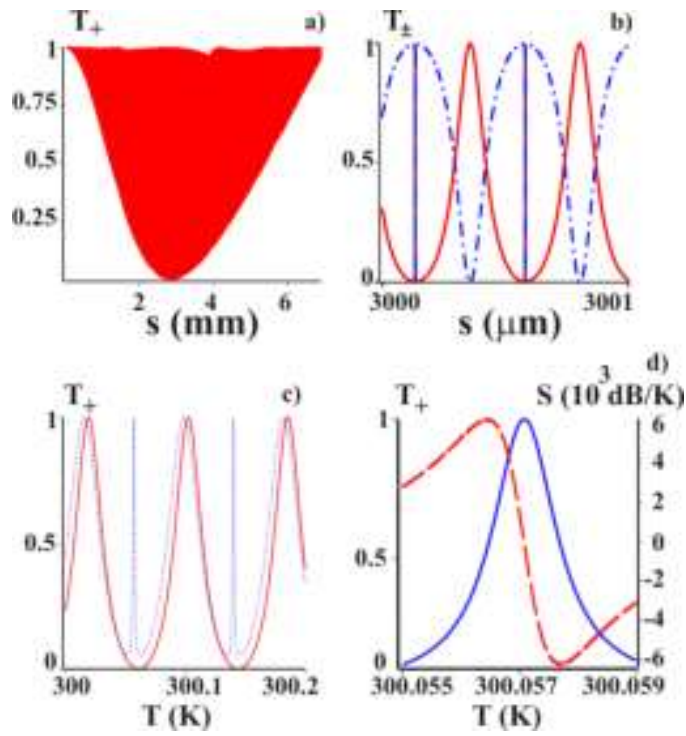

Figure 2. Transmission of $|1, R\rangle$ OV vs coupling segment's length: wavelength-scale oscillations (b) on the background of large-scale variations (a); red curve is for $T_{+}$(in input power units).

Temperature control of OV conversion for larger (c) and smaller (d) temperature variations. In (d) solid blue curve is for $T_{+}$and red dashed one is for sensitivity $S$. Parameters: $s=s_{0}=2,86 \mathrm{~mm}$; (c) -

$$
d=8,44 \mathrm{~cm},(\mathrm{~d})-d=84,4 \mathrm{~cm} \text {. For the blue dotted curve in (c): } s=0.8 s_{0} .
$$

This property might be also useful for sensor applications. Indeed, Figure $2 \mathrm{c}$ shows a temperature dependence of the transmission coefficient. This curve also features slow and rapid variations; temperature ranges with rapid variations (Figure 2d) feature higher sensitivity $S \equiv d\left(10 \lg T_{+}\right) / d T, T$ being temperature, at the cost of decreased operation range. It is helpful to note that although the power $T_{+}$of the $|1, R\rangle$ component may vary due to instability of the input power, the ratio $T_{+} / T_{-}$is independent of such external power variations.

An acknowledged property of fiber resonators is a sharp increasing of the field intensity in the resonance points. For LRs on multimode fibers this is also the case: Figure 3a shows sharp peaks on $\Lambda_{+}$curve accompanied with small-scale variations of $T_{+}$. The same is also true for $\Lambda_{-}$(not shown). The peak magnitudes are closely correlated with the fastness of variations of $T_{+}$. This can be explained as follows. The resonance points correspond to zeroes of the denominators of $R_{i}$, which gives four sets of resonance parameters $d$ and $s$ :

$$
\begin{gathered}
\mathrm{s}_{m}^{1,2}=\pi \delta \beta_{1,2}^{-1}(2 m+0.5), d_{n m}^{1,2}=\pi \tilde{\beta}_{l}^{-1}(2 n+1.5)-\mathrm{s}_{m}^{1,2}, \\
\tilde{\mathrm{s}}_{m}^{1,2}=\pi \delta \beta_{1,2}^{-1}(2 m+1.5), \tilde{d}_{n m}^{1,2}=\pi \tilde{\beta}_{l}^{-1}(2 n+0.5)-\mathrm{s}_{m}^{1,2},
\end{gathered}
$$

where $n, m=0, \pm 1, \pm 2 \ldots$ (provided $d>\mathrm{s}>0$ ). For example, near the resonance points $\mathrm{s}_{m}$ one has $\mathrm{s} \approx \mathrm{s}_{m}+\varepsilon, \varepsilon<<\mathrm{s}_{m}$, and:

$$
\tan \left(\Phi_{1}-\Phi_{2}\right) \square \frac{\lambda}{\varepsilon \pi n_{c o}} \cot ^{2}\left(\frac{\pi}{4}-\frac{\kappa_{2}}{2}\right) \equiv \frac{\xi}{\varepsilon} .
$$

The characteristic length $\xi$ determines the narrowness of spikes on $T_{+}$curve. Simultaneously, one obtains $\Lambda_{ \pm}=\frac{1}{4} \tan ^{2}\left(\frac{\pi}{4}-\frac{\kappa_{2}}{2}\right) \square \frac{\lambda}{\xi}$, which proves the observed correlation. The ratio $\lambda / \xi$ rather chaotically depends on the resonance point's order $m$ (Figure 3b) and, in principle, can be arbitrary large, which opens possibilities 
for increasing sensitivity. Such correlated behavior of $T_{+}$and $\Lambda_{ \pm}$is characteristic to resonance phenomena. However, here we have a special type of the resonance associated with TC flipping, at which the output power vanishes only in, say, $|1, R\rangle$-component, being redistributed into $|2, R\rangle$-component.

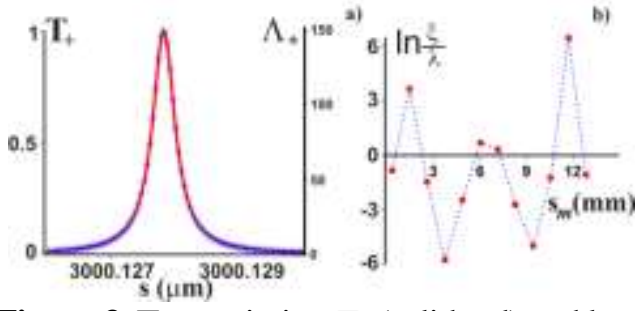

Figure 3. Transmission $T_{+}$(solid red) and loop ("starred" blue) $\Lambda_{+}$power spikes (a) at the resonance point $s=s_{0}$, in input power units (note identity of the forms of the curves); (b) characteristic length as function of resonance length's $s_{m}$ order. LR's parameters as in Figure 2.
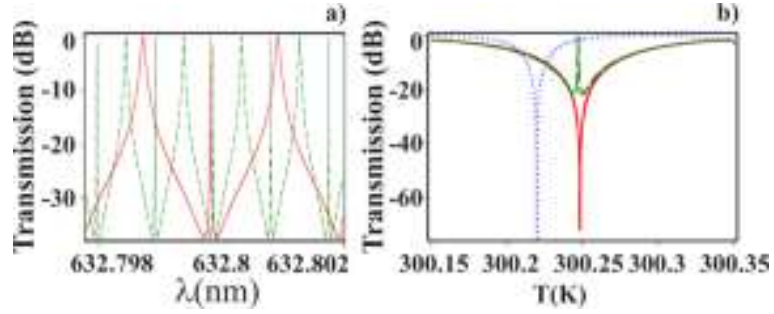

Figure 4. (a) Transmission $T_{+}$vs wavelength for LRs with $d=8,44 \mathrm{~cm}$ (solid red curve) and $d=25,33 \mathrm{~cm}$ (dashed green curve); $s=s_{5}=4,855 \mathrm{~cm}$. (b) Transmission vs temperature; green dashed curve: $d=8,44 \mathrm{~cm}, s=0,88 s_{5}$; solid red curve: $d=8.44 \mathrm{~cm}, s=s_{5}$; dotted blue curve $d=$ $42,22 \mathrm{~cm}, s=s_{5}$.

If the LR is excited with the Hermite-Gaussian (HG) modes $H G^{e v, o d} \propto|1, L\rangle \pm|2, L\rangle$, then $L_{1}=1, L_{2}= \pm 1$ and from (9) one can ascertain that the HG modes are not changed by the LR, that is, they are eigenmodes of the LR with eigenvalues $\exp \left(i \Phi_{1,2}\right)$. Power spikes in the loop can be useful for nonlinear applications. Using the same procedure as while obtaining (12) one can get for the field in the loop near resonances $\mathrm{s}_{m}^{1,2}$ (upper signs) and $\tilde{\mathrm{s}}_{m}^{1,2}$ : $\mid$ loop $\rangle \propto I_{\mp}(|1, L\rangle \pm|2, L\rangle)$. In this way, in the resonances the loop field is given either by even or odd HG modes, that is by the LR's eigenmodes. One should stress that studying effects concerned with power variation of the outcoming OV implies the ability to separate this OV from the complex outcoming field. This task can be fulfilled using effective existing techniques [34].

Finally, consider wavelength dependence of LR's transmission. Figure 4a shows typical comb filter-like dependence with increasing comb's thickness for values of the loop length. One can estimate here formal parameters of the LR. For two types of $\mathrm{s}_{m}$ and $\tilde{\mathrm{s}}_{m}$ resonance points (narrow and wide peaks) for both loop lengths the Q-factors are of the order $10^{8} \div 10^{7}$, which of the standard performances for such systems. FSR has the order of $0,001 \mathrm{~nm}$. A more detailed consideration seems redundant unless any assumptions on losses are made. Drawing analogies with [5] one can expect that this property of transmission curves can be also used for sensing application. Figure $4 \mathrm{~b}$ shows that near the resonances LRs exhibit impressive sensitivity to temperature variations. It is seen that performances of the LR are very sensitive to the deviation from the resonance point. Of course, for more realistic models that take account of power losses one expects less impressive characteristics.

\section{Conclusion}

In conclusion, in this paper we have theoretically studied the transmission of OVs through the lossless LR on multimode fibers. We have shown that by changing the length of the loop and coupling area one can control the weights of OVs with opposite TCs in the outcoming field. We have shown that in the resonance points such weights are sensitive to wavelength and ultra-subwavelength variations of the LR's parameters, in this way enabling super-efficient TC and OAM control. We have also discussed sensor application of this effect. We have demonstrated the existence of power spikes in the loop at the resonance, which might be useful for nonlinear applications. We have also studied the spectral characteristics of OV transmission in LRs and shown feasibility of comb filters on the basis of the effect of OV's power control.

\section{Acknowledgments}

This work was supported by the V.I. Vernadsky Crimean Federal University Development Program for 2015-2024 (Grant № VG02/2020). 
Секция: Компьютерная оптика и нанофотоника

Propagation of optical vortices in loop resonators on the basis of multimode optical fibers

\section{References}

[1] Stokes, L.F. All-single-mode fiber resonator / L.F. Stokes, M. Chodorow, H.J. Shaw // Opt. Let. - 1982. - Vol. 7. - P. 288-230.

[2] $\mathrm{Yu}, \mathrm{W}$. A tunable all-fiber filter based on microfiber loop resonator / W. Yu, Z. Xu, H. Changlun, B. Jian, Y. Guoguang // Appl. Phys. Let. - 2008. - Vol. 92. - P. 191112.

[3] Madamopoulos, N. Fiber loop mirror based single-platform multifunctional Michelson-GiresTournois filter // Opt. Commun. - 2019. - Vol. 436. - P. 134-142.

[4] Harun, S.W. Microfiber loop resonator based temperature sensor / S.W. Harun, K.S. Lim, S.S.A. Damanhuri, H. Ahmad // JEOS:RP. - 2011. - Vol. 6. - P. 11026.

[5] Sumetsky, M. The microfiber loop resonator: Theory, experiment, and application / M. Sumetsky, Y. Dulashko, J.M. Fini, A. Hale, D.J. DiGiovanni // J. Lightw. Technol. - 2006. Vol. 24. - P. 242-250.

[6] Linslal, C.L. Analysis and modeling of an optical fiber loop resonator and an evanescent field absorption sensor for the application for chemical detection / C.L. Linslal, P.M. Syam Mohan, A. Halder, T.K. Gangopadhyay // Sens. Actuator A Phys. - 2013. - Vol. 194. - P. 160-168.

[7] Xu, F. Demonstration of a refractometric sensor based on optical microfiber coil resonator / F. Xu, G. Brambilla // Appl. Phys. Let. - 2008. - Vol. 92. - P. 101126.

[8] Wang, Q. Research on fiber loop coupled resonator slow light and displacement sensing technology / Q. Wang, X. Feng, Y. Zhao, J. Li, H. Hu // Sens. Actuator A Phys. - 2015. - Vol. 233. - P. 472-479.

[9] Lee, T. Resonantly enhanced third harmonic generation in microfiber loop resonators / T. Lee, N.G.R. Broderick, G. Brambilla // J. Opt. Soc. Am. B. - 2013. - Vol. 30. - P. 505-511.

[10] Gouveia, M.A. Second harmonic generation and enhancement in microfibers and loop resonators / M.A. Gouveia, T. Lee, R. Ismaeel, M. Ding, N.G.R. Broderick, C.M.B. Cordeiro, G. Brambilla // Appl. Phys. Let. - 2013. - Vol. 102. - P. 201120

[11] Kowsari, A. Dynamic analysis of optical microfiber coil resonators / A. Kowsari, V. Ahmadi, G. Darvish, M.K. Moravvej-Farshi // Appl. Opt. - 2016. - Vol. 55. - P. 6680-6687.

[12] Li, J.-H. Versatile hybrid plasmonic microfiber knot resonator / J.-H. Li, J.-H. Chen, S.-C. Yan, Y.-P. Ruan, F. Xu, Y.-Q. Lu // Opt. Let. - 2017. - Vol. 42. - P. 3395-3398.

[13] Ramelow, S.Strong nonlinear coupling in a $\mathrm{Si}_{3} \mathrm{~N}_{4}$ ring resonator / S. Ramelow, A. Farsi, Z. Vernon, S. Clemmen, X. Ji, J.E. Sipe, M. Liscidini, M. Lipson, A.L. Gaeta // Phys. Rev. Lett. 2019. - Vol. 122. - P. 153906

[14] Fülöp, A. High-order coherent communications using mode-locked dark-pulse Kerr combs from microresonators / A. Fülöp, M. Mazur, A. Lorences-Riesgo, Ó.B. Helgason, P.-H. Wang, Y. Xuan, D.E. Leaird, M. Qi, P.A. Andrekson, A.M. Weiner, V. Torres-Company // Nat. Commun. - 2018. - Vol. 9. - P. 1598

[15] Sumetsky, M. Optical microfiber coil delay line // Opt. Express. - 2009. - Vol. 17. - P. 71967205.

[16] Liu, D. Submicron-resonator-based add-drop optical filter with an ultra-large free spectral range / D. Liu, C. Zhang, D. Liang, D. Dai // Opt. Express. - 2019. - Vol. 27. - P. 416-422.

[17] Armaroli, A. Microwave generation on an optical carrier in microresonator chains / A. Armaroli, P. Féron, Y. Dumeige // Phys. Rev. A. - 2018. - Vol. 98. - P. 013848.

[18] Leykam, D. Reconfigurable topological phases in next-nearest-neighbor coupled resonator lattices / D. Leykam, S. Mittal, M. Hafezi, Y.D. Chong // Phys. Rev. Lett. - 2018. - Vol. 121. P. 023901

[19] Jing, H. Nanoparticle sensing with a spinning resonator / H. Jing, H. Lü, S.K. Özdemir, T. Carmon, F. Nori // Optica. - 2018. - Vol. 5. - P. 1424-1430.

[20] Liu, M. Graphene-decorated microfiber knot as a broadband resonator for ultrahigh-repetitionrate pulse fiber lasers / M. Liu, R. Tang, A.-P. Luo, W.-C. Xu, Z.-C. Luo // Photon. Res. - 2018. - Vol. 6. - P. C1-C7.

[21] Padgett, M.J. Orbital angular momentum 25 years on // Opt. Express. - 2017. - Vol. 25. - P. 11265-11274. 
[22] Ramachandran, S. Optical vortices in fiber / S. Ramachandran, P. Kristensen // Nanophotonics. - 2013. - Vol. 2. - P. 455-474.

[23] Yavorsky, M.A. Revised model of acousto-optic interaction in optical fibers endowed with a flexural wave / M.A. Yavorsky, D.V. Vikulin, E.V. Barshak, B.P. Lapin, C.N. Alexeyev // Opt. Let. - 2019. - Vol. 44. - P. 598-601.

[24] Yavorsky, M.A. Polarization-dependent orbital angular momentum flipping in fibers with acousto-optic interaction / M.A. Yavorsky, D.V. Vikulin, E.V. Barshak, B.P. Lapin, C.N. Alexeyev // J. Phys. Conf. Ser. - 2019 (in press).

[25] Yavorsky, M.A. All-fiber polarization-dependent optical-vortex-controlling via acousto-optic interaction / M.A. Yavorsky, D.V. Vikulin, E.V. Barshak, B.P. Lapin, C.N. Alexeyev // Proceedings of the International Conference Days on Diffraction. - 2019 (in press).

[26] Barshak, E.V. Polarization and topological mode dispersion of optical vortices in circular optical fibers / E.V. Barshak, M.A. Yavorsky, D.V. Vikulin, B.P. Lapin, A.V. Volyar, C.N. Alexeyev // Computer Optics. - 2019. - Vol. 43(1). - P. 25-34. DOI: 10.18287/2412-61792019-43-1-25-34.

[27] Lapin, B. Transmission of optical vortices through Bragg optical multihelicoidal fibers of heterogeneous type / B. Lapin, M. Yavorsky, E. Barshak, D. Vikulin, C. Alexeyev // J. Phys. Conf. Ser. - 2019. - Vol. 1368. - P. 022021.

[28] Turpin, A. Engineering of orbital angular momentum supermodes in coupled optical waveguides / A. Turpin, G. Pelegrí, J. Polo, J. Mompart, V. Ahufinger // Sci. Rep. - 2017. Vol. 7. - P. 44057.

[29] Zhang, Z. Low-crosstalk orbital angular momentum fiber coupler design / Z. Zhang, J. Gan, X. Heng, M. Li, J. Li, S. Xu, Z. Yang // Opt. Express. - 2017. - Vol. 28. - P. 11200-11209.

[30] Alexeyev, C.N. Inversion of the topological charge of optical vortices in a coil fiber resonator / C.N. Alexeyev, A.V. Milodan, M.C. Alexeyeva, M.A. Yavorsky // Opt. Let. - 2016. - Vol. 41. - P. 1526-1529.

[31] Alexeyev, C.N. Transmission of optical vortices through fiber loop resonators / C.N. Alexeyev, E.V. Barshak, B.P. Lapin, M.A. Yavorsky // Opt. Let. - 2019. - Vol. 44. - P. 4044-4047.

[32] Alexeyev, C.N. Higher order modes of coupled optical fibers / C.N. Alexeyev, N.A. Boklag, M.A. Yavorsky // J. Opt. - 2010. - Vol. 12. - P. 115704.

[33] Snyder, A.W. Optical Waveguide Theory /A.W. Snyder, J.D. Love - London, New York: Chapman and Hall, 1985. - 750 p.

[34] Huang, H. Mode division multiplexing using an orbital angular momentum mode sorter and MIMO-DSP over a graded-index few-mode optical fibre / H. Huang, G. Milione, M.P.J. Lavery, G. Xie, Y. Ren, Y. Cao, N. Ahmed, T.A. Nguyen, D.A. Nolan, M.-J. Li, M. Tur, R.R. Alfano, A.E. Willner // Sci. Rep. - 2015. - Vol. 5. - P. 14931. 